\title{
Clinical significance of miR-195 in hepatocellular carcinoma and its biological function in tumor progression
}

This article was published in the following Dove Medical Press journal: OncoTargets and Therapy

\author{
Xiaoyan Chen' \\ Angang Wang ${ }^{2}$ \\ 'Department of Laboratory Medicine, \\ Women and Children's Hospital of \\ Linyi City, Shandong 276000, People's \\ Republic of China; ${ }^{2}$ Department \\ of Laboratory Medicine, People's \\ Hospital of Yutai County, Shandong \\ 272300, People's Republic of China
}

Correspondence: Xiaoyan Chen Department of Laboratory Medicine, Women and Children's Hospital of Linyi City, No I, South Qinghe Road, Shandong 276000, People's Republic of China

Tel/fax +86539321 6147

Email ling1987kang@163.com
Background: Hepatocellular carcinoma (HCC) is one of the most lethal cancer types all over the world. Chronic viral hepatitis B and hepatitis $\mathrm{C}$ are risk factors that are associated with the development of HCC. The aim of this study is to identify the diagnostic role of serum miR-195 in HCC.

Patients and methods: The expression levels of miR-195 were detected in $120 \mathrm{HCC}$ patients, 64 hepatitis only patients, and 118 healthy control as well as 4 HCC cell lines, by using quantitative real-time PCR. The association of miR-195 with clinicopathological parameters of patients was analyzed with the chi-squared test. The receiver operating characteristic (ROC) curve was adopted to estimate the potential diagnostic value of miR-195. The cell experiments were carried out to verify the functional role of miR-195.

Results: The expression of miR-195 was downregulated in HCC cells and serum of patients compared to the controls (all $P<0.05$ ). The miR-195 expression was associated with lymph node metastasis and TNM stage. The ROC curve analysis showed that miR-195 may be a noninvasive diagnostic marker for patients. By using miR-195 mimic or inhibitor, cell proliferation, migration, and invasion were inhibited by miR-195 overexpression but promoted by reduced expression of miR-195.

Conclusion: The downregulation of miR-195 may serve as a novel diagnostic biomarker for differentiating HCC patients, healthy individuals, and hepatitis patients, and may involve in the tumor progression of HCC.

Keywords: miRNA-195, diagnosis, proliferation, migration, invasion, hepatocellular carcinoma

\section{Introduction}

Hepatocellular carcinoma (HCC) is one of the most common primary liver malignancy, with high mortality worldwide. The development of HCC is thought to be multifactorial, and seem to be closely related to chronic hepatitis B virus (HBV), hepatitis C virus infections. ${ }^{1-4}$ Most HCC patients are usually asymptomatic at an early stage, leading to initial diagnosis at an advanced stage with shorter overall survival. ${ }^{5,6}$ Surgical resection is the main treatment for $\mathrm{HCC}$ at present. Although multimodal treatment of $\mathrm{HCC}$ has made significant progress, the prognosis of HCC patients remains unsatisfactory due to high recurrence and early distant metastasis. ${ }^{7,8}$ Thus, special and effective cancer-related biomarkers for early diagnosis of HCC from healthy individuals, as well as from hepatitis patients are urgently required.

miRNAs are small endogenous noncoding RNAs of $\sim 22$ nucleotides in length, which regulate gene expression by binding to $3^{\prime}$ UTR of target mRNA to initiate cleavage or translational repression of target transcripts..$^{9,10}$ Numerous studies have 
shown that miRNAs played important roles in biological processes, such as cell differentiation, proliferation, migration, invasion, apoptosis, and tumor metastasis. ${ }^{11,12}$ miRNAs can be oncogenes or tumor suppressor genes in a majority of cancer during tumorigenesis, which are considered as novel biomarkers for tumor diagnosis and therapy. miR-195 has been reported to be downregulated in the tissues of HCC patients. ${ }^{13,14}$ However, the expression pattern of miR-195 in HCC cell lines and serum of HCC patients is still elusive.

In this study, we detected the expression of miR-195 in the serum of HCC patients, hepatitis only patients, and healthy individuals, as well as in cell lines. Its diagnostic value for cancer patients was also assessed. In addition, we investigated the effects of miR-195 on biological behaviors of HCC cells.

\section{Patients and methods}

\section{Patients and specimens}

From June 2012 to July 2017, a total of 120 HCC patients, 64 hepatitis only patients, and 118 healthy individuals at the People's Hospital of Yutai County were recruited into this study. Blood samples were collected during the $\mathrm{HCC}$ diagnosis or physical examination. A total of $10 \mathrm{~mL}$ of whole blood was collected from each participant and the serum was separated from whole blood by centrifugation, and then stored at $-80^{\circ} \mathrm{C}$ until RNA extraction. All the patients had not received any treatment before sampling. The clinicopathological information from all the HCC patients was collected and is summarized in Table 1. The protocols of this study were approved by the Ethics Committee of the People's Hospital of Yutai County and adhered to the tenets of the Declaration of Helsinki. All the participants signed the written informed consent.

\section{Cell lines and transfection}

Human normal liver epithelial cell (THLE3) and HCC cell lines (HepG2 and SK-Hep1) were purchased from the American Type Culture Collection (ATCC), Manassas, VA, USA. HCC cell lines (Huh7 and PLC) were purchased from China Center for Type Culture Collection (CCTCC, Hubei, China). All cells were cultured in DMEM (Thermo Fisher Scientific, Waltham, MA, USA) with $10 \% \mathrm{FBS}$ at $37^{\circ} \mathrm{C}$ in a humid incubator with $5 \% \mathrm{CO}_{2}$. HCC cells were seeded into 6 -well plates at a density of $2 \times 10^{5}$ and incubated 24 hours, and then transfected with miR-195 mimic, inhibitor, or their negative control (NC) using Lipofectamine 2000 reagent (Thermo Fisher Scientific) following the instructions of the manufacturer. Untreated cells were used as blank control.
Table I The relationship between miR-I95 expression and clinicopathological parameters of HCC patients

\begin{tabular}{|c|c|c|c|c|}
\hline \multirow[t]{2}{*}{ Parameters } & \multirow{2}{*}{$\begin{array}{l}\text { No. of } \\
\text { cases } \\
(n=\mid 20)\end{array}$} & \multicolumn{2}{|c|}{$\begin{array}{l}\text { miR- } 195 \\
\text { expression }\end{array}$} & \multirow[t]{2}{*}{$P$-value } \\
\hline & & $\begin{array}{l}\text { Low } \\
(n=67)\end{array}$ & $\begin{array}{l}\text { High } \\
(n=53)\end{array}$ & \\
\hline Gender & & & & 0.415 \\
\hline Male & 98 & 53 & 45 & \\
\hline Female & 22 & 14 & 8 & \\
\hline Age (years) & & & & 0.449 \\
\hline$\geq 55$ & 59 & 35 & 24 & \\
\hline$<55$ & 61 & 32 & 29 & \\
\hline Tumor size $(\mathrm{cm})$ & & & & 0.293 \\
\hline$\geq 5$ & 72 & 43 & 29 & \\
\hline$<5$ & 48 & 24 & 24 & \\
\hline Hepatitis history & & & & 0.922 \\
\hline Yes & 64 & 36 & 28 & \\
\hline No & 56 & 31 & 25 & \\
\hline AFP (ng/mL) & & & & 0.183 \\
\hline$\geq 20$ & 58 & 36 & 22 & \\
\hline$<20$ & 62 & 31 & 31 & \\
\hline Lymph node metastasis & & & & 0.040 \\
\hline Positive & 51 & 34 & 17 & \\
\hline Negative & 69 & 33 & 36 & \\
\hline Differentiation & & & & 0.159 \\
\hline Poor & 63 & 39 & 24 & \\
\hline Moderate + well & 57 & 28 & 29 & \\
\hline TNM stage & & & & 0.030 \\
\hline I-II & 48 & 21 & 27 & \\
\hline III-IV & 72 & 46 & 26 & \\
\hline
\end{tabular}

Abbreviations: AFP, $\alpha$-fetoprotein; HCC, hepatocellular carcinoma.

\section{RNA isolation and quantitative real-time (qRT)-PCR analysis}

Total RNA was isolated from serum and cell lines by using Trizol reagent (Thermo Fisher Scientific) according to the manufacturer's instruction. Reverse transcription was performed using miScript (SYBR ${ }^{\mathrm{TM}}$ ) Green PCR kit (Qiagen NV, Venlo, the Netherlands) according to the manufacturer's protocol. Total RNA concentration was assessed by measuring absorbance at 260 and $280 \mathrm{~nm}$ (A 260/280 ratio) and checked by gel electrophoresis individually. In general, $800 \mathrm{ng}$ of RNA from $1 \mathrm{~mL}$ of serum was used for further use. The relative expression levels of miR-195 were detected by qRT-PCR, which was performed with the SYBR ${ }^{\mathrm{TM}}$ Green PCR master mix (Thermo Fisher Scientific) on an ABI 7900 real-time PCR system (Thermo Fisher Scientific). The relative quantification of miR-195 expression was calculated with the $2^{-\Delta \Delta C t}$ methods normalized to miR- 16 .

\section{Cell proliferation assay}

The viability of HCC cells was measured with the colorimetric MTT assay. Cells were plated in 96-well plates $\left(1 \times 10^{4}\right.$ cells per well $)$ and transfected with miR-195 mimic, 
inhibitor, or their NC. After transfection, 48 hours later, the medium was added with $20 \mu \mathrm{L}$ MTT solution $(5 \mathrm{mg} / \mathrm{mL}$; Sigma-Aldrich Co., St Louis, MO, USA) every 24 hours and incubated for 4 hours at $37^{\circ} \mathrm{C}$. The supernatant was discarded, and $100 \mu \mathrm{L}$ dimethyl sulfoxide (Sigma-Aldrich Co.) was added to dissolve formazan crystals. Cell proliferation was determined using the colorimetric analysis (wavelength, $490 \mathrm{~nm}$ ) by a spectrophotometer (Multiskan MK3; Thermo Fisher Scientific) at 24, 48, 72, and 96 hours. Each treatment group was measured at least three times.

\section{Cell migration and invasion assays}

Cell migration and invasion assays were assessed with 24-well Transwell chamber $(8-\mu \mathrm{m}$ pore size, Multiskan MK3; Thermo Fisher Scientific). For invasion assay, the top chamber was coated with Matrigel (BD biosciences, Bedford, MA, USA) on the upper side. Hundred microliter cell suspension with $1 \times 10^{5}$ cells were plated in the top chamber with serum-free DMEM at $37^{\circ} \mathrm{C}$ for 24 hours. The bottom chamber was filled with $600 \mu \mathrm{L}$ DMEM containing 20\% FBS. Then non-invading cells on the upper surface were removed using cotton-tipped swabs, while the cells that invaded the lower side of the membrane were fixed in $3.7 \%$ formaldehyde for 5 minutes and stained with $0.1 \%$ crystal violet for 15 minutes. The cells were counted using a microscope (Olympus Corporation, Tokyo, Japan). For migration assay, the same procedure was followed as with invasion assay except that no Matrigel was used and the permeating time was 12 hours.

\section{Statistical analysis}

All statistical analyses were performed using the SPSS 21.0 software (IBM Corporation, Armonk, NY, USA) and GraphPad Prism 5.0 software (GraphPad Software, Inc., La Jolla, CA, USA). Data are presented as the mean \pm SD. The significance between groups was analyzed by Student's $t$-test or one-way ANOVA. The relationship between the expression of miR-195 and clinicopathological features was analyzed by the chi-square $\left(\chi^{2}\right)$ test. The receiver operating characteristic (ROC) curves were generated to evaluate the diagnostic potential of serum miR-195 by calculating the area under the ROC curve (AUC). Each experiment was repeated at least three times. A $P$-value $<0.05$ was considered to be of statistical significance.

\section{Results}

\section{Expression of miR-195 in the serum of HCC patients and HCC cell lines}

A previous study by Sohn et al have shown that the serum levels of miR-195 were lower in HCC patients than in patients with chronic HBV. ${ }^{15}$ To further investigate the roles of miR-195 in the development of HCC, we detected the expression levels of miR-195 in the serum samples of HCC patients (64 HCC patients with hepatitis and $56 \mathrm{HCC}$ patients without hepatitis), hepatitis only patients, and healthy control, as well as in the cell lines, using qRT-PCR. As shown in Figure 1A, the relative serum expression of miR-195 was significantly downregulated in HCC patients $(1.355 \pm 0.46)$ compared to healthy control $(2.430 \pm 0.83)(P<0.001)$. There is no significant difference between healthy control and hepatitis only patients $(2.341 \pm 0.75)(P>0.05)$. Compared to healthy control, the relative expression levels of miR-195 were significantly lower in HCC patients without hepatitis $(1.371 \pm 0.48)$ (Figure 1B, $P<0.001)$. The result of Figure 1C showed that the relative expression of miR-195 was significantly lower in HCC patients with hepatitis $(1.340 \pm 0.45)$ than in hepatitis only patients $(P<0.001)$. These results suggest miR-195 may have diagnostic value in distinguishing HCC patients from healthy individuals, as well as from hepatitis patients. Meanwhile, compared to the normal liver epithelial cell THLE3, the expression levels of miR-195 were decreased in HCC cell lines (all $P<0.001$, Figure 1D).

\section{The relationship between miR-I95 expression and clinicopathological characters of HCC patients}

To investigate whether miR-195 expression was involved in the development of HCC, we analyzed the relationship between its expression and patients' clinicopathological features. We divided the HCC patients into a low-expression group ( $n=67)$ and high-expression group $(n=53)$ according to the mean value of miR-195 expression level (1.355). As shown in Table 1, the expression of miR-195 was significantly associated with lymph node metastasis $(P=0.040)$ and TNM stage $(P=0.030)$. However, there was no significant relationship with other parameters, such as gender, age, tumor size, hepatitis history, $\alpha$-fetoprotein (AFP), and differentiation (all $P>0.05$ ).

\section{Diagnostic value of miR-195 for HCC}

We plotted the ROC curve to assess the potential usefulness of serum miR-195 as a noninvasive biomarker for the early diagnosis of HCC from healthy individuals, as well as from hepatitis patients. As shown in Figure 2A, the AUC value was 0.876 (95\% CI $=0.817-0.907)$. Using the optimum cutoff value of 1.685 according to the Youden index, the sensitivity and specificity were $76.7 \%$ and $77 \%$, respectively. The miR-195 was effective in differentiating HCC patients from healthy individuals with high specificity and 
A
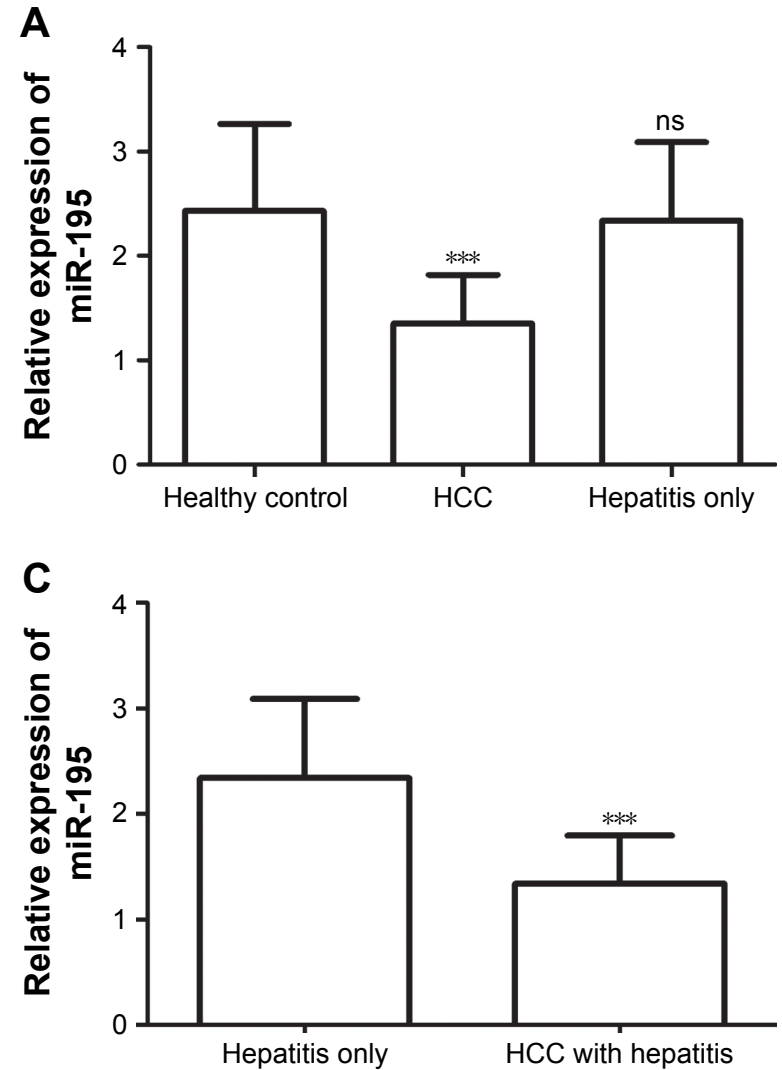

B

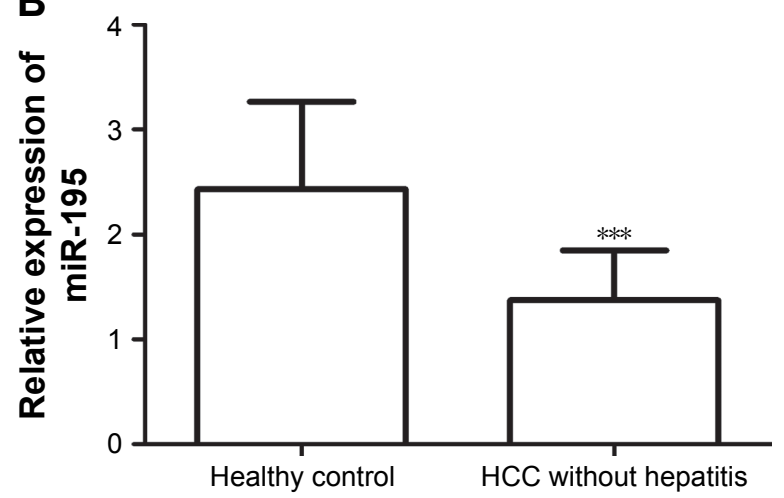

D

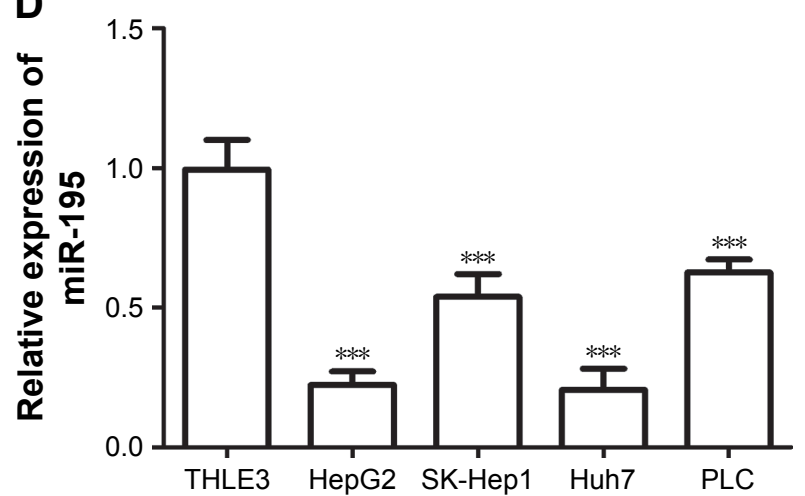

Figure I Expression of miR-195 was determined by qRT-PCR, and the miR-16 was used as an internal control.

Notes: (A) The serum expression of miR-195 was downregulated in HCC patients compared to healthy control (***P<0.00I). There is no significant difference between healthy control and hepatitis only patients (ns $P>0.05$ ). (B) The serum expression of miR- 195 was lower in $\mathrm{HCC}$ patients without hepatitis than healthy control $(* * * P<0.00 \mathrm{I}$ ). (C) The serum expression of miR-195 was lower in HCC patients with hepatitis than hepatitis patients (***P<0.00I). (D) The relative expression of miR-I95 was decreased in $\mathrm{HCC}$ cell lines compared to the normal liver epithelial cell $(* * * P<0.001)$.

Abbreviations: HCC, hepatocellular carcinoma; qRT, quantitative real-time; ns, no statistical significance.

sensitivity (Figure 2A). The AUC value was 0.859 (95\% $\mathrm{CI}=0.805-0.913)$ in Figure $2 \mathrm{~B}$, which suggests miR-195 can also distinguish HCC patient without hepatitis from healthy individuals yielding the sensitivity of $71.4 \%$ and specificity of $74.7 \%$ at the optimum cutoff value of 1.665 . Results in Figure 2C showed that miR-195 expression had a higher accuracy in differentiating $\mathrm{HCC}$ patients from hepatitis patients based on AUC of 0.868 (95\% CI $=0.806-0.930)$.
A

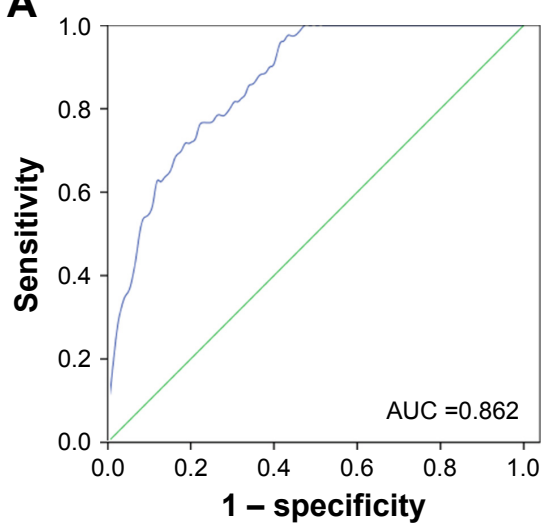

B

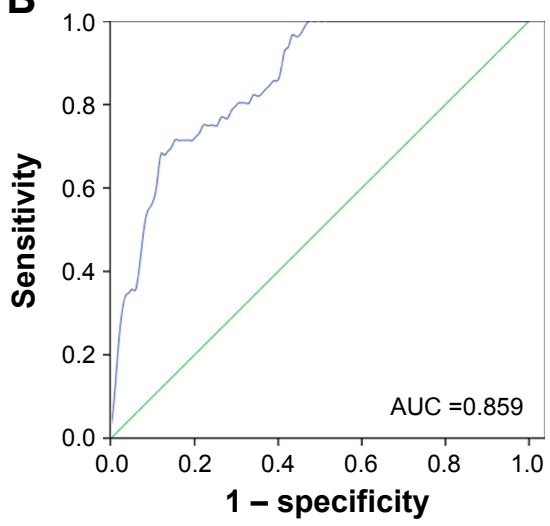

C

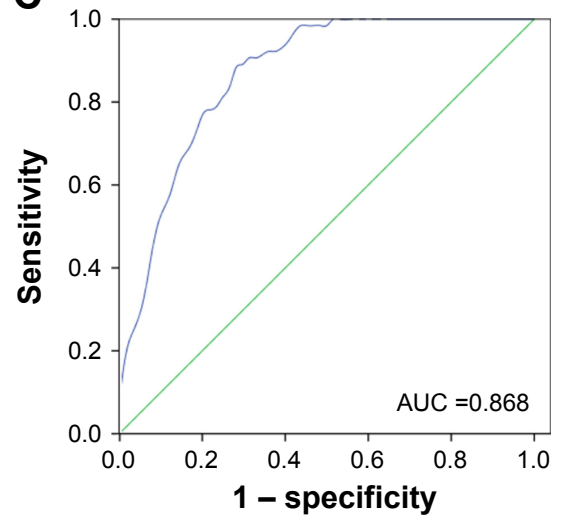

Figure 2 Diagnostic value of miR-195 for HCC by establishing a ROC curve.

Notes: (A) ROC curve of the diagnostic value of miR-195 in differentiating HCC patients from healthy individuals. The AUC is 0.862 , with a sensitivity of $76.7 \%$ and a specificity of $77.0 \%$ at the optimum cutoff value of 1.685 . (B) ROC curve of the diagnostic value of miR-195 in distinguishing HCC patients without hepatitis from healthy individuals. The AUC is 0.859 , with a sensitivity of $71.4 \%$ and a specificity of $74.7 \%$ at the optimum cutoff value of 1.450 . (C) ROC curve of the diagnostic value of miR-195 in distinguishing HCC patients with hepatitis from hepatitis patients based on AUC of 0.868 . The sensitivity and specificity were $89.1 \%$ and $71.9 \%$, respectively, at the optimum cutoff value of 1.960

Abbreviations: AUC, area under the ROC curve; HCC, hepatocellular carcinoma; ROC, receiver operating characteristic. 
In addition, the sensitivity and specificity for serum miR-195 were $89.1 \%$ and $71.9 \%$, respectively, at the cutoff point of 1.960. Taken together, miR-195 can differentiate HCC patients, healthy individuals, and hepatitis patients.

\section{Effects of miR-195 on HCC cell proliferation, migration, and invasion}

To investigate the functional role of miR-195 in HCC, the effects of miR-195 on HCC cell proliferation was performed in vitro. We transfected miR-195 mimic or miR-195 inhibitor, or their NC into HepG2 and Huh7 cells, which exhibit a low miR-195 expression. Then, we analyzed miR-195 expression levels after transfection by qRT-PCR and found that the expression of miR-195 was significantly increased in the cells transfected with miR-195 mimic but decreased in those transfected with miR-195 inhibitor, compared to the controls (all $P<0.01$, Figure 3A). To examine the role of miR-195 in the proliferation of HCC cells, MTT assay was performed. The results showed that cell proliferation of $\mathrm{HepG} 2$ and Huh7 cells transfected with miR-195 mimic was significantly lower but in those transfected with miR-195 inhibitor was higher, compared to their controls ( $P<0.05$, Figure 3B). In addition, Transwell assays were employed to evaluate the impact of miR-195 on migration and invasion. The results showed that miR-195 mimic inhibited the migration and invasion, but miR-195 inhibitor promoted the migration and invasion, compared to their controls (all $P<0.05$, Figure 4). Taken together, these results demonstrate that miR-195 inhibits HCC cell proliferation, migration, and invasion.

\section{Discussion}

The detection of HCC at an early stage have a relatively longer survival rate, which can significantly impact curative treatment. ${ }^{16}$ Currently, the diagnosis of $\mathrm{HCC}$ is mainly based on assessing serum AFP levels, computed tomography detection, and magnetic resonance imaging. ${ }^{17,18}$ However, a lower rate of early diagnosis and lack of effective biological indicators for monitoring tumor occurrence may be the
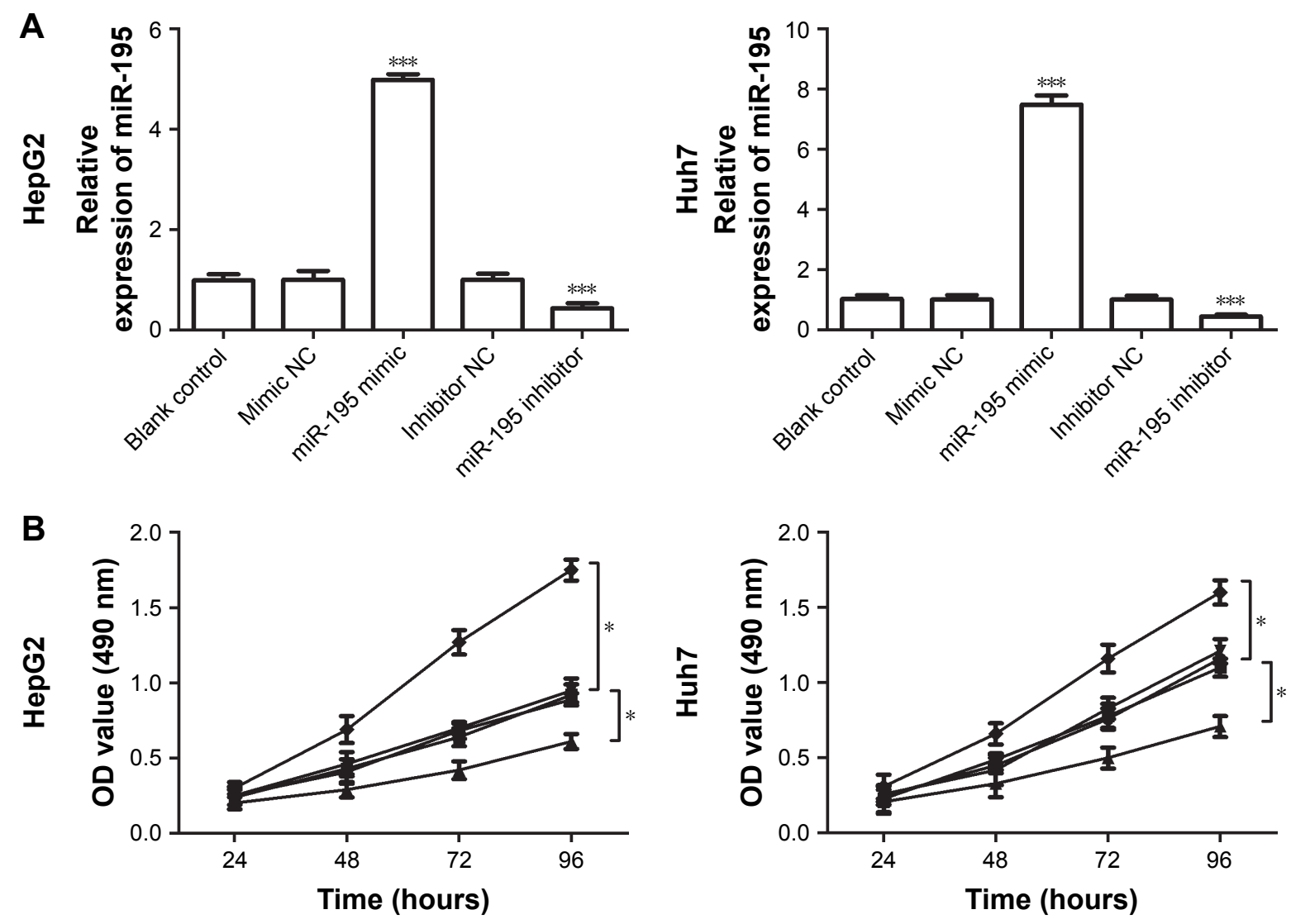

Blank control

tr Mimic NC

miR-195 mimic

$\rightarrow$ Inhibitor NC

$\sim$ miR-195 inhibitor

Figure 3 Effects of miR-195 on cell proliferation in HepG2 and Huh7 cells.

Notes: The cells were transfected with miR-195 mimic, inhibitor, or their NCs. (A) The expression of miR-195 was significantly increased in cells transfected with miR-195 mimic and decreased in cells transfected with miR-195 inhibitor. (B) The proliferation ability was significantly reduced by miR-195 mimic but was promoted by the miR-195 inhibitor $(* P<0.05$, and $* * * P<0.001)$.

Abbreviations: NC, negative control; OD, optical density. 

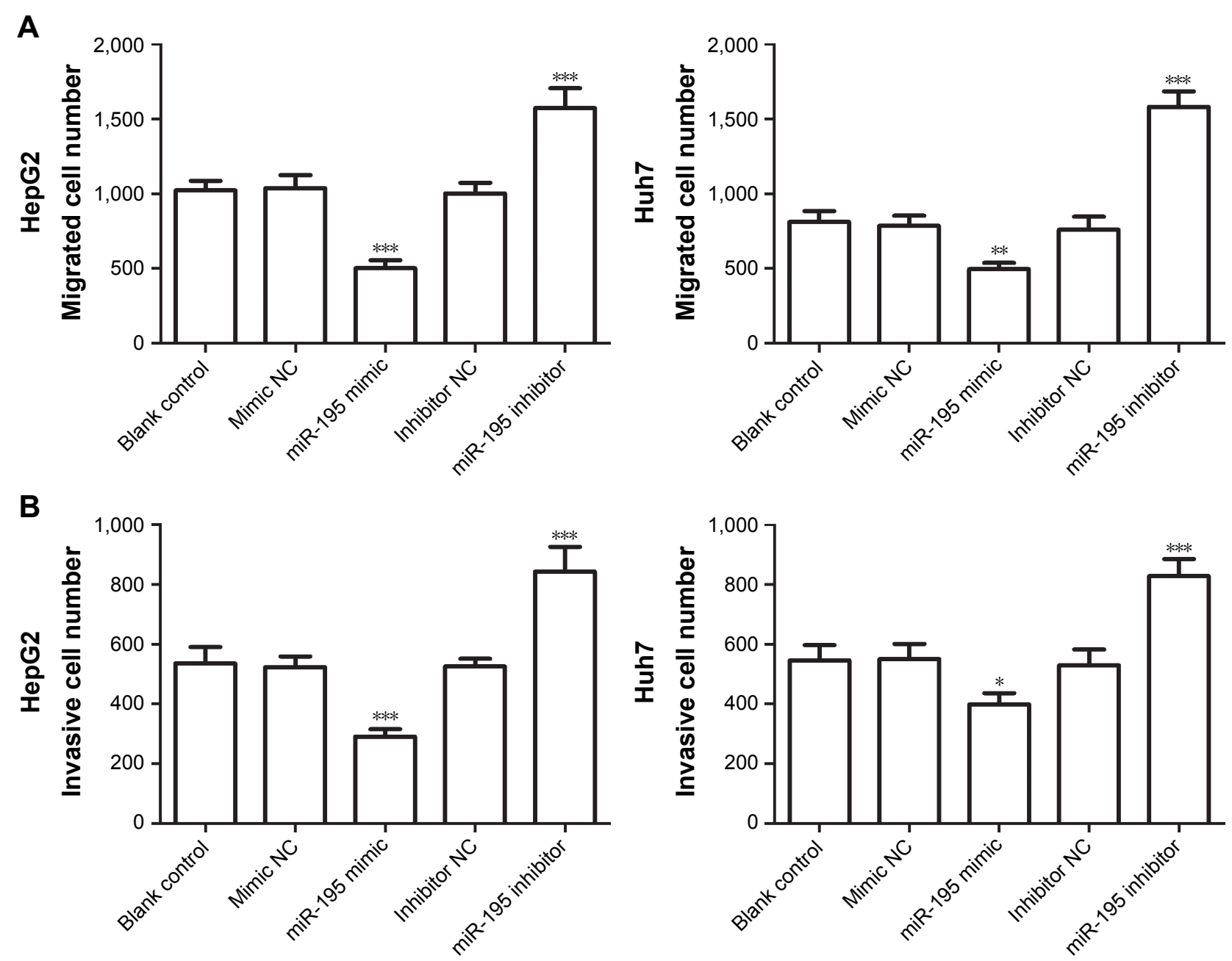

Figure 4 Cell migration and invasion were performed by Transwell or Matrigel precoated Transwell assay in HepG2 and Huh7 cells.

Notes: (A and B) The results indicated that, compared to controls, overexpression of miR-195 by miR-195 mimic could inhibit the cell migration and invasion, but the downregulation of miR-195 expression could promote the cell migration and invasion $(* P<0.05, * * P<0.0 \mathrm{I}$, and $* * * P<0.00 \mathrm{I})$.

current major problem for poor prognosis of HCC. Thus, the noninvasive sensitive markers for early HCC detection are needed.

miRNAs dysregulation has been observed in cancer research, which serves as potential biomarkers of diagnosis and prognosis as well as therapeutic targets for cancers. ${ }^{19-23}$ Numerous studies have proven that miRNAs play a regulatory effect on the wide aspects of biological processes, such as cell proliferation, migration, and invasion. ${ }^{24,25}$ In HCC, some miRNAs have also been identified as dysregulated in tissues or serum samples. ${ }^{26-28}$ For instance, Suehiro et al analyzed serum exosomal miR-122 and miR-21 expression levels in HCC patients that underwent transarterial chemoembolization (TACE), demonstrating that the exosomal miR-122 level alterations may represent a predictive biomarker in $\mathrm{HCC}$ patients with liver cirrhosis treated with TACE. ${ }^{28}$ A study by Yoon et al found that miR-21 expression was significantly increased in HCC tissues compared to nontumor tissues, and pointed that elevated miR-21 expression might represent a biomarker for HCC prognosis. ${ }^{29}$ Overexpression of miR-26b was found by $\mathrm{Li}$ et al inhibited the proliferation, invasion, and migration of HCC cells by targeting EphA2. ${ }^{30}$ Taken together, these studies demonstrated that the cancer-related miRNAs and corresponding direct target genes are essential for HCC processes.

In this study, by using qRT-PCR, we detected the expression of miR-195 in the serum of HCC patients, hepatitis patients, and healthy control, as well as in HCC cell lines. The results showed that miR-195 significantly decreased in the serum of HCC patients compared to healthy control. The serum expression levels of miR-195 were lower in HCC patients with hepatitis than the hepatitis patients, and lower in HCC patients without hepatitis than healthy individuals. Similarly, the expression of miR-195 was downregulated in 
HCC cell lines compared to the normal liver epithelial cell (THLE3). The analysis results of the correlation between miR-195 expression and clinicopathological characteristics showed that the miR-195 expression was significantly associated with lymph node metastasis and TNM stage. However, no significant relationship was found with other parameters, such as gender, age, tumor size, hepatitis history, AFP, and differentiation. The results showed miR-195 expression has no relationship with hepatitis history. Taken together, the results indicated that miR-195 may act as a suppressor gene and involve in the development of HCC. Then by using ROC curve analysis, we further analyzed the potential diagnostic value of miR-195 in HCC. The higher AUC results and promising sensitivity and specificity showed miR-195 had a relatively high accuracy in differentiating $\mathrm{HCC}$ patients from healthy individuals, as well as in differentiating HCC patients from hepatitis patients, which indicated miR-195 may be a diagnostic biomarker for HCC.

In addition to HCC, the role of miR-195 was studied in other malignancies, such as gastric cancer, breast cancer, colon cancer, and cholangiocarcinoma (CCA). ${ }^{31-34} \mathrm{Li}$ et al indicated that miR-195 expression was downregulated in colon cancer tissues and associated with the enhanced proliferation, migration, and invasion of colon cancer, which is a potential diagnostic marker and therapeutic target for improving the clinical management of colon cancer. ${ }^{33}$ The study in CCA by Chen et al revealed that miR-195 expression was significantly lower in CCA patients than in normal control, and it was reference prognosis marker of CCA. ${ }^{34}$ These previous studies showed that miR-195 plays a crucial role in the progression of cancers. Previous study by Wang et al verified that miR-195 is a vital regulator in the lung metastasis of HCC by using a lung metastasis HCC cell line BEL-7402. ${ }^{13}$ The results of Yan et al's study showed the expression of miR-195 is downregulated in HCC tissues and cell lines, and miR-195 inhibits cell proliferation via targeting AEG-1 in HCC. ${ }^{14}$ These studies suggested that miR-195 is associated with progression of HCC. To verify the functional role of miR-195 in HCC, the effects of miR-195 on HCC cell proliferation, migration, and invasion were explored by regulating miR-195 expression using miR-195 mimic or inhibitor. The results indicated that, compared to controls, miR-195 mimic obviously inhibited the cell proliferation, migration, and invasion, conversely, miR-195 inhibitor promoted biological behaviors in both HepG2 and Huh7 cells, which confirmed the functional role of miR-195 in HCC. In a previous study, Li et al identified LncRNA UCA1 enhanced mitochondrial function and cell viability through the miR-195/ARL2 signaling pathway in bladder cancer, and provided UCA1 as a potential diagnosis and treatment target for bladder cancer. ${ }^{35}$ However, the potential molecular mechanism of miR-195 in the processes of HCC remain unconfirmed in our present study. Further studies are needed to confirm the role of miR-195 in HCC progression.

\section{Conclusion}

Our data in this study revealed that the downregulated expression of miR-195 may serve as a potential noninvasive diagnostic biomarker in differentiating HCC patients from healthy individuals, as well as from hepatitis patients. miR-195 is associated with the development of HCC and may be involved in the tumor progression of $\mathrm{HCC}$, and has the potential to be used in the targeted therapy for HCC patients.

\section{Disclosure}

The authors report no conflicts of interest in this work.

\section{References}

1. Iavarone M, Colombo M. HBV infection and hepatocellular carcinoma. Clin Liver Dis. 2013;17(3):375-397.

2. Xu C, Zhou W, Wang Y, Qiao L. Hepatitis B virus-induced hepatocellular carcinoma. Cancer Lett. 2014;345(2):216-222.

3. Rongrui L, Na H, Zongfang L, Fanpu J, Shiwen J. Epigenetic mechanism involved in the $\mathrm{HBV} / \mathrm{HCV}$-related hepatocellular carcinoma tumorigenesis. Curr Pharm Des. 2014;20(11):1715-1725.

4. Singh AK, Kumar R, Pandey AK. Hepatocellular carcinoma: causes, mechanism of progression and biomarkers. Curr Chem Genom Transl Med. 2018;12(1):9-26.

5. Gramenzi A, Tedeschi S, Cantarini MC, et al. Outcome of hepatocellular carcinoma in human immunodeficiency virus-infected patients. Dig Liver Dis. 2013;45(6):516-522.

6. Ganslmayer M, Hagel A, Dauth W, et al. A large cohort of patients with hepatocellular carcinoma in a single European centre: aetiology and prognosis now and in a historical cohort. Swiss Med Wkly. 2014; 144:w13900.

7. Graf D, Vallböhmer D, Knoefel WT, et al. Multimodal treatment of hepatocellular carcinoma. Eur J Intern Med. 2014;25(5):430-437.

8. Moray G, Kirnap M, Akdur A, et al. Outcomes of patients with hepatocellular carcinoma after liver transplant. Exp Clin Transplant. 2015 13(Suppl 3):30-32.

9. van Rooij E. The art of microRNA research. Circ Res. 2011;108(2): 219-234.

10. Shukla GC, Singh J, Barik S. MicroRNAs: processing, maturation, target recognition and regulatory functions. Mol Cell Pharmacol. 2011; 3(3):83-92.

11. Miller N, Bédard YC, Cooter NB, Shaul DL. Histological changes in the genital tract in transsexual women following androgen therapy. Histopathology. 1986;10(7):661-669.

12. Anfossi S, Fu X, Nagvekar R, Calin GA. MicroRNAs, regulatory messengers inside and outside cancer cells. Adv Exp Med Biol. 2018; 1056:87-108.

13. Wang M, Zhang J, Tong L, Ma X, Qiu X. miR-195 is a key negative regulator of hepatocellular carcinoma metastasis by targeting FGF2 and VEGFA. Int J Clin Exp Pathol. 2015;8(11):14110-14120.

14. Yan JJ, Chang Y, Zhang YN, Lin JS, He XX, Huang HJ. miR-195 inhibits cell proliferation via targeting AEG-1 in hepatocellular carcinoma. Oncol Lett. 2017;13(5):3118-3126. 
15. Sohn W, Kim J, Kang SH, et al. Serum exosomal microRNAs as novel biomarkers for hepatocellular carcinoma. Exp Mol Med. 2015; 47(9):e184.

16. Tsuchiya N, Sawada Y, Endo I, Saito K, Uemura Y, Nakatsura T. Biomarkers for the early diagnosis of hepatocellular carcinoma. World J Gastroenterol. 2015;21(37):10573-10583.

17. Choi J, Kim GA, Han S, Lee W, Chun S, Lim YS. Longitudinal assessment of three serum biomarkers to detect very early stage hepatocellular carcinoma. Hepatology. Epub 2018 Aug 28.

18. Aghoram R, Cai P, Dickinson JA; Cochrane Hepato-Biliary Group. Alpha-foetoprotein and/or liver ultrasonography for screening of hepatocellular carcinoma in patients with chronic hepatitis B. Cochrane Database Syst Rev. 2012;9:CD002799.

19. Cheng G. Circulating miRNAs: roles in cancer diagnosis, prognosis and therapy. Adv Drug Deliv Rev. 2015;81:75-93.

20. Kavitha N, Vijayarathna S, Jothy SL, et al. MicroRNAs: biogenesis, roles for carcinogenesis and as potential biomarkers for cancer diagnosis and prognosis. Asian Pac J Cancer Prev. 2014;15(18):7489-7497.

21. Bertoli G, Cava C, Castiglioni I. MicroRNAs: new biomarkers for diagnosis, prognosis, therapy prediction and therapeutic tools for breast cancer. Theranostics. 2015;5(10):1122-1143.

22. Svoronos AA, Engelman DM, Slack FJ. OncomiR or tumor suppressor? The duplicity of microRNAs in cancer. Cancer Res. 2016;76(13): 3666-3670.

23. Schwarzenbach H. Clinical relevance of circulating, cell-free and exosomal microRNAs in plasma and serum of breast cancer patients. Oncol Res Treat. 2017;40(7-8):423-429.

24. Jiang Y, Zhang M, Guo T, Yang C, Zhang C, Hao J. MicroRNA-21-5p promotes proliferation of gastric cancer cells through targeting SMAD7. Onco Targets Ther. 2018;11:4901-4911.

25. Zhu L, Chen Y, Nie K, Xiao Y, Yu H. miR-101 inhibits cell proliferation and invasion of pancreatic cancer through targeting STMN1. Cancer Biomark. 2018;23(2):301-309.
26. Callegari E, Elamin BK, Sabbioni S, Gramantieri L, Negrini M. Role of microRNAs in hepatocellular carcinoma: a clinical perspective. Onco Targets Ther. 2013;6:1167-1178.

27. Li L, Jia L, Ding Y. Upregulation of miR-375 inhibits human liver cancer cell growth by modulating cell proliferation and apoptosis via targeting ErbB2. Oncol Lett. 2018;16(3):3319-3326.

28. Suehiro T, Miyaaki H, Kanda Y, et al. Serum exosomal microRNA-122 and microRNA-21 as predictive biomarkers in transarterial chemoembolization-treated hepatocellular carcinoma patients. Oncol Lett. 2018;16(3):3267-3273.

29. Yoon JS, Kim G, Lee YR, et al. Clinical significance of microRNA-21 expression in disease progression of patients with hepatocellular carcinoma. Biomark Med. 2018;12(10):1105-1114.

30. Li H, Sun Q, Han B, Yu X, Hu B, Hu S. miR-26b inhibits hepatocellular carcinoma cell proliferation, migration, and invasion by targeting EphA2. Int J Clin Exp Pathol. 2015;8(5):4782-4790.

31. Zhang W, Wang Y, Zhu Z, Zheng Y, Song B. Propofol inhibits proliferation, migration and invasion of gastric cancer cells by up-regulating microRNA-195. Int J Biol Macromol. 2018;120(Pt A):975-984.

32. Marques MM, Evangelista AF, Macedo T, et al. Expression of tumor suppressors miR-195 and let-7a as potential biomarkers of invasive breast cancer. Clinics (Sao Paulo). 2018;73(12):e184.

33. Li B, Wang S, Wang S. miR-195 suppresses colon cancer proliferation and metastasis by targeting WNT3A. Mol Genet Genomics. 2018; 293(5):1245-1253.

34. Chen Q, Wang C, Zhang H, et al. Expression levels of serum miRNA-195 in different types of patients with cholangiocarcinoma and its value to determine the prognosis thereof. Oncol Lett. 2018;15(4):5947-5951.

35. Li HJ, Sun XM, Li ZK, et al. LncRNA UCA1 promotes mitochondrial function of bladder cancer via the miR-195/ARL2 signaling pathway. Cell Physiol Biochem. 2017;43(6):2548-2561.
OncoTargets and Therapy

\section{Publish your work in this journal}

OncoTargets and Therapy is an international, peer-reviewed, open access journal focusing on the pathological basis of all cancers, potential targets for therapy and treatment protocols employed to improve the management of cancer patients. The journal also focuses on the impact of management programs and new therapeutic agents and protocols on

\section{Dovepress}

patient perspectives such as quality of life, adherence and satisfaction The manuscript management system is completely online and includes a very quick and fair peer-review system, which is all easy to use. Visit http://www.dovepress.com/testimonials.php to read real quotes from published authors. 